available for part-time training particularly at the SHO/Registrar level. Indeed, I have reluctantly come to the conclusion that the problems facing trainee psychiatrists who have young children are more formidable than they were a generation ago, as I trained on a part-time basis.

I deplore the potential loss to psychiatry of motivated recruits whose time spent in child rearing provides an experience which is far from irrelevant to any branch of psychiatry. I hope, in collaboration with the Dean, to arrange a meeting where the implications of part-time training and other related issues can be discussed and measures taken to provide a remedy.

ANN Gath Registrar

\section{Computerised audit systems}

DEAR SIRS

I read with interest of the computerised audit system currently under development by Professor Marks (Psychiatric Bulletin, August 1989, 14, 495) and would like to echo his comments that such systems make the process of audit easier. SafetyNet is now being established in several hospitals across the UK and provides a sophisticated but simple solution to monitoring/charting clinical progress (employing recognised 5 point scales) and staff interventions. Since the system was first outlined in the Psychiatric Bulletin $(13,677-679)$ it has been enhanced and now incorporates a module called ResearchBase (also written using Ashton Tate's Dbase IV).

ResearchBase (which is also available independently of SafetyNet) allows any user (without computer expertise) to add in rating scales and questionnaires for time-series data collection. The system handles non-branching and branching scales, all data types (data are automatically validated) and will be useful to anyone wishing to repeatedly administer schedules as part of a research project, or who wishes to speedily develop a custom built audit or case register system that collects the data and descriptions they have selected. The system can handle virtually unlimited patient numbers, and up to 99 separate scales each with up to 999 questions.

Once data are on the system they can be very simply transferred to SPSS PC + (SPSS UK) for analysis. ResearchBase comes with complete ICD-9 codes and descriptors (around 5,000) and medication codes and descriptors (proper drug names drawn from the BNF). Both SafetyNet and ResearchBase run on standalone IBM compatible machines (AT 286 or 386 computers with hard disks running MSDos) and do not require Dbase IV. Anyone inter- ested in SafetyNet or ResearchBase can write to me at 309 Gray's Inn Road, for more information.

SafetyNet

JASON TAYLOR

\section{Gray's Inn Road}

London WCIX $8 Q F$

\section{Discharge summaries}

DeAr Sirs

We were interested to read of the brief computerised discharge summary reported by Wattis \& Protheroe (Psychiatric Bulletin, June 1990, 14, 330), in particular that $93 \%$ of GP's questioned preferred a short summary with the opportunity to request more detail.

For the last 12 months our psychogeriatric department has kept detailed case summaries based on Institute of Psychiatry guidelines and including DSM-IIIR diagnoses. One copy is filed in the case notes, a second on the ward to provide instant access should patients be re-admitted and case notes not immediately available, and, a third in the department for internal audit and reference. The GP is sent a prose style letter on one side of A4 with a note explaining that a more detailed account of the patient's illness, history and progress is available on request.

Since this has been the policy we have discharged 90 patients to 72 GPs (about $50 \%$ of those in the catchment area) and, as yet, nobody has asked for the detailed summary.

Studies of GPs' requirements of discharge letters suggest the brief style is most popular (Craddock \& Craddock, 1989; Kerr, 1990) and although a proportion always claim to want more detailed information our experience is that this is not requested even when the offer is clearly made.

A standard computerised format will become essential for audit but there is a danger that using too brief a summary will result in oversimplified audit results that may not accurately reflect our workload and clinical dilemmas and we feel the more detailed summaries will provide a more useful database for this purpose. As Craddock \& Craddock (1989) demonstrated, the requirements of GPs and psychiatrists differ.

D. N. ANDERSON A. J. KIRBY

Fazakerley Hospital

Longmoor Lane

Liverpool $L 97 A L$

\section{References}

Craddock, N. \& Craddock, B. (1989) Psychiatric discharge summaries: differing requirements of psychiatrists 